\title{
Reduction of sawtooth amplitude by resonant magnetic perturbation
}

\author{
Jianchao $\mathrm{Li}^{1,2}$, Yonghua Ding 3 , Qingquan $\mathrm{Yu}^{4}$, Nengchao $\mathrm{Wang}^{3}$, Da Li ${ }^{3}$, Xiaoqing Zhang ${ }^{3}$, Dongliang $\mathrm{Han}^{3}$, \\ Zhipeng Chen ${ }^{3}$, Zhoujun Yang ${ }^{3}$, Song Zhou ${ }^{3}$, Wei Yan ${ }^{3}$, Yunfeng Liang ${ }^{3,5,6}$, Xiaolong Zhang ${ }^{1,2}$, Xiaodong Lin², \\ Huibin Sun ${ }^{1,2}$, Xiang Gao ${ }^{1,6}$, Jiangang $\mathrm{Li}^{1,6}$ and the J-TEXT team ${ }^{7}$
}

\begin{abstract}
${ }^{1}$ Advanced Energy Research Center, Shenzhen University, Shenzhen 518060, People's Republic of China
${ }^{2}$ Key Laboratory of Optoelectronic Devices and Systems of Ministry of Education and Guangdong Province, College of Optoelectronic Engineering, Shenzhen University, Shenzhen 518060

${ }^{3}$ International Joint Research Laboratory of Magnetic Confinement Fusion and Plasma Physics, State Key Laboratory of Advanced Electromagnetic Engineering and Technology, School of Electrical and Electronic Engineering, Huazhong University of Science and Technology, Wuhan, 430074, China

${ }^{4}$ Max-Planck-Institut für Plasmaphysik, D-85748 Garching, Germany

${ }^{5}$ Forschungszentrum Jülich GmbH, Institut für Energie- und Klimaforschung_Plasmaphysik, 52425 Jülich, Germany

${ }^{6}$ Institute of Plasma Physics, Chinese Academy of Sciences, P.O. Box 1136, Hefei, Anhui 330031, People’s Republic of China

${ }^{7}$ See the author list of Yunfeng Liang et al Nucl. Fusion 59, 112016(2019)
\end{abstract}

E-mail: yhding@mail.hust.edu.cn, qingquan.yu@ipp.mpg.de

\begin{abstract}
Sawtooth crashes of large amplitude are detrimental to tokamak plasma confinement, and the amplitude has to be reduced for a fusion reactor. A novel method, i.e., applying $m / n=2 / 2$ resonant magnetic perturbations (RMPs), is found to be able to reduce the sawtooth magnitude in J-TEXT tokamak experiments. The penetration of RMPs and formation of 2/2 locked island are found to be related to the reduction of sawtooth magnitude. With the available 2/2 RMP field, the sawtooth mitigation is reproducibly achieved with $q_{\mathrm{a}}<2.8$ and $n_{\mathrm{e}}<2 \times 10^{19} \mathrm{~m}^{-3}$ in J-TEXT.
\end{abstract}

Keywords: sawtooth, resonant magnetic perturbation, 2/2 island, tokamak plasma

\section{I introduction}

Sawtooth instability [1-4] is one of the most violent magnetohydrodynamic (MHD) instabilities which has to be actively controlled in a tokamak fusion reactor. Large sawtooth crashes can trigger neoclassical tearing modes (NTMs) [5] to cause 
the degradation of plasma confinement and even major disruptions. On the other hand, small sawtooth crashes are beneficial to the exhaust of helium ash and impurities in the plasma core [6]. Hence, maintaining the sawtooth magnitude at a moderate level is essential for fusion plasmas. Substantial researches have been devoted to the sawtooth control, mainly by applying high power radio-frequency waves [7-10] or neutral beam injection (NBI) [11-12]. However these methods rely on accurate power deposition around the surface where the safety factor $q=1$ or inside it, being complicated from the technology point of view for a reactor. It is desirable to find a simple method for sawtooth amplitude mitigation.

Externally applied resonant magnetic perturbations (RMPs) have been applied to control MHD instabilities, such as NTMs [13], resistive wall modes [14], and edge localized modes [15]. However, existing research efforts haven't shown promising potentials of RMPs in sawtooth control yet. The $n=1$ RMPs had been applied in DIII-D and RFX experiments, and the sawtooth amplitude was reduced when the coupling between the plasma response to the $m / n=2 / 1 \mathrm{RMP}$ and the internal kink mode was sufficiently strong [16] $(\mathrm{m} / \mathrm{n}$ is the poloidal /toroidal mode number). The sawtooth mitigation in steady state had been achieved only with a low edge safety factor, $q_{95}<2$, which is not realistic for a fusion reactor.

Unlike the $n=1 \mathrm{RMP}$, the $n=2 \mathrm{RMP}$ could be promising in sawtooth control based on the following considerations: (i) the $2 / 2 \mathrm{RMP}$ is able to induce a $2 / 2$ magnetic island at $q=1$ surface to change the local plasma pressure and current density gradient and therefore the $1 / 1$ mode stability; (ii) the $2 / 2$ island can act as a secondary island to affect the sawtooth crash [17]; (iii) the RMP spectrum can have a dominant 2/2 component but little 2/1 component, allowing the sawtooth control for $q_{\mathrm{a}}>2$. In this work, we present the first experimental demonstration of sawtooth mitigation by applying the $2 / 2$ RMP on J-TEXT tokamak. Section II describes the experimental set-up, the experimental results are depicted in Section III, and the last section is discussion and summary.

\section{Experimental set-up}

J-TEXT is a tokamak with a major radius $R=1.05 \mathrm{~m}$ and minor radius $a=0.255 \mathrm{~m}$ [18]. The RMP system consists of 24 in-vessel saddle coils [19-20]. By feeding currents to them as shown in FIG. 1(a1), they can generate $n=2$ RMPs with a dominant resonant $2 / 2$ component of an amplitude of 7.5 Gauss $/ \mathrm{kA}$ at plasma edge $(r=a)$ and the amplitudes of $m / n$ $=3 / 2,4 / 2$ and 5/2 sidebands are 1.6, 1.0 and 0.8 Gauss/kA, respectively. With this configuration mode, the RMP system can produce the maximal $2 / 2$ component while a small 2/1 component $(0.5$ Gauss/kA) for avoiding triggering the $2 / 1$ locked mode.

In this work, the electron temperature is measured by 24-channel heterodyne electron cyclotron emission (ECE) radiometer in Port 4 [21], providing the spatial profile of the electron temperature with the spatial resolution of $\sim 2 \mathrm{~cm}$ on the middle plane with toroidal magnetic field 1.74T. The center electron temperature and toroidal rotation velocity are detected by a tangential x-ray imaging crystal spectrometer (XICS) by analyzing emission of Ar XVII in plasma core [22]. A laser-based far-infrared polarimeter-interferometer system (Polaris) has been implemented for electron density profile with radius resolution of $3 \mathrm{~cm}$ [23]. The upgraded magnetic diagnostic of Mirnov probe arrays on the J-TEXT contains one poloidal array with 48 probes and two toroidal arrays with 25 probes, which is used for analysis of poloidal and toroidal mode numbers [24]. In this paper, the soft X-ray emission is measured by the soft X-ray diagnostic system (SXR) in Port 
3 , containing 3 cameras with a radial resolution of $\sim 1.5 \mathrm{~cm} \mathrm{[25].}$

\section{Experimental results}

For the experimental results described in this section, the plasma parameters are as follows unless otherwise mentioned: major/minor radius $\left(R_{0} / a\right)$ is $1.05 \mathrm{~m} / 0.255 \mathrm{~m}$, plasma current $I_{\mathrm{p}}=210 \mathrm{kA}$, toroidal magnetic field $B_{\mathrm{t}}=1.75 \mathrm{~T}$, and the safety factor $q \approx 2.6$ at the plasma edge. The core line-averaged electron density at $R=1.05 \mathrm{~m}$ is in the range of $1.2-1.8 \times 10^{19}$ $\mathrm{m}^{-3}$.

FIG. 1 displays a typical example of sawtooth control by applying the $n=2$ RMPs. The target plasma has an initial line averaged electron density $n_{\mathrm{e} 0}$ of $1.2 \times 10^{19} \mathrm{~m}^{-3}$, core electron temperature $T_{\mathrm{e}, 0}$ of around $1.0 \mathrm{keV}$ measured by XICS. Without applying RMPs, sawtooth period $\tau_{\mathrm{st}}$ is around $1.6 \mathrm{~ms}$, and its magnitude $A_{\mathrm{st}}$ is around 0.16 , where $A_{\mathrm{st}}$ is defined by $\Delta T_{\mathrm{e}, \mathrm{st}} / T_{\mathrm{e}}$, and $\Delta T_{\mathrm{e}, \mathrm{st}}$ is the ECE signal variation during sawtooth crash measured at $r=3.5 \mathrm{~cm}$. The RMP coil current, $I_{\mathrm{RMP}}$, is first ramped up quickly to $2 \mathrm{kA}$ from a time $0.31 \mathrm{~s}$ and then slowly to $4 \mathrm{kA}$, keeping at $4 \mathrm{kA}$ till $0.405 \mathrm{~s}$ (FIG. 1(a)).

Three types of sawtooth responses to the $n=2$ RMPs are identified from the ECE signal measured near the $q=1$ surface (FIG. 1(a) green line) and the sawtooth period $\tau_{\text {st }}$ (FIG. 1(b)), marked as region I, II and III. Both $\tau_{\text {st }}$ and $A_{\text {st }}$ are unaffected for $I_{\mathrm{RMP}}<3 \mathrm{kA}$ in region I. Once $I_{\mathrm{RMP}}>3 \mathrm{kA}$, there is a small increase of $\tau_{\mathrm{st}}$ and $A_{\mathrm{st}}$ in region II. Further increasing $I_{\mathrm{RMP}}$ to be above $3.85 \mathrm{kA}$ at $0.348 \mathrm{~s}, \tau_{\mathrm{st}}$ decreases from $2.2 \mathrm{~ms}$ to $1 \mathrm{~ms}$ within two sawtooth periods, and $A_{\mathrm{st}}$ also decreases, showing a bifurcation from region II to III. The toroidal rotation velocity of Argon ions $\left(\mathrm{Ar}^{16+}\right)$ in the core is about $30-50$ $\mathrm{km} / \mathrm{s}$ in the counter- $I_{\mathrm{p}}$ direction before applying RMPs, and it changes towards the co- $I_{\mathrm{p}}$ direction with a variation $\Delta V_{\Phi} \sim 0$, $\sim 16$ and $\sim 26 \mathrm{~km} / \mathrm{s}$ in region I, II and III, respectively (FIG. 1(c)). When $I_{\mathrm{RMP}}$ is reduced to 0 around $0.418 \mathrm{~s}, \tau_{\mathrm{st}}\left(\right.$ and $A_{\mathrm{st}}$ ) recovers from $1.1 \mathrm{~ms}$ to $2.2 \mathrm{~ms}$ (from 0.10 to 0.21 ), and $\Delta V_{\Phi}$ decreases to $\sim 0$ after $20 \mathrm{~ms}$.

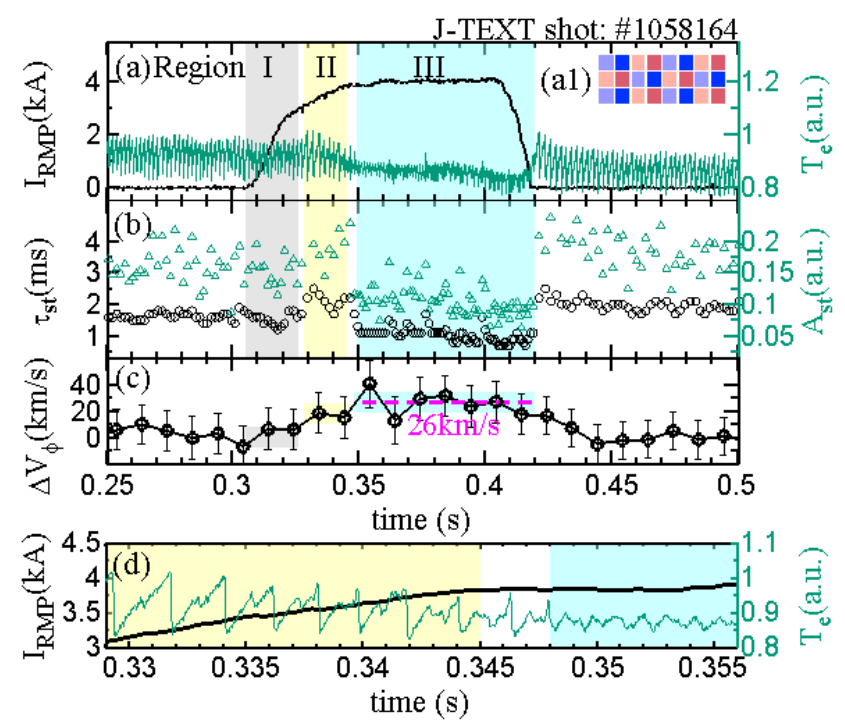

FIG. 1. Control of sawteeth by $2 / 2$ RMP in discharge \#1058164. Time evolution of: (a) RMP coil current $I_{\text {RMP }}$ and ECE signal measured inside $q=1$ rational surface, (b) sawtooth period ( $\tau_{\mathrm{st}}$, black circles) and magnitude ( $A_{\mathrm{st}}$, green triangles) calculated from the ECE signal in the plasma core at $r=3.5 \mathrm{~cm}$, (c) toroidal rotation velocity of $\mathrm{Ar}^{16+}$ in plasma core, (d) expanded view of (a). (a1) shows the distribution of currents in 24 RMP coils, where red (blue) represents $2(-2) \mathrm{kA} *$ turn, and the light red (light blue) represents $1(-1) \mathrm{kA} *$ turn. 


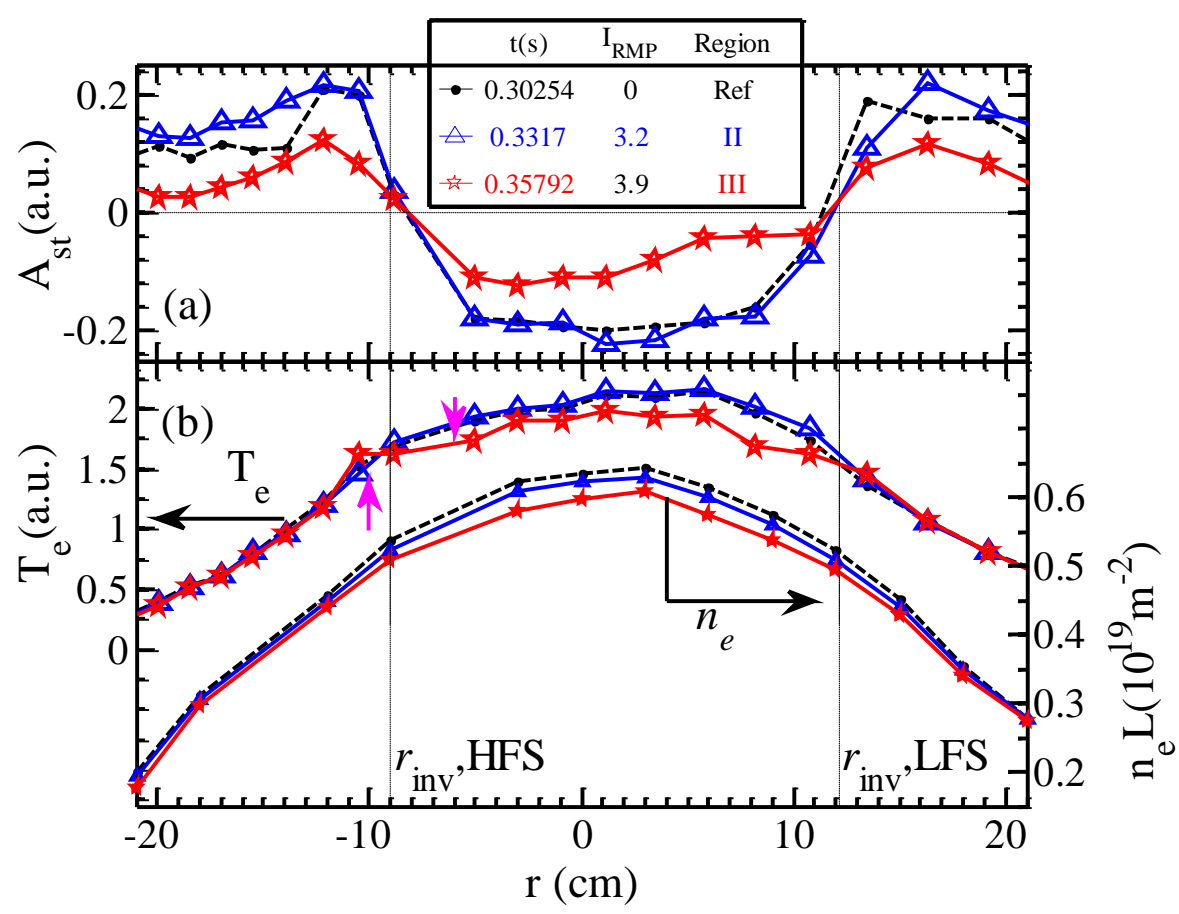

FIG. 2. Radial profiles of (a) $A_{\mathrm{st}}$, (b) $T_{\mathrm{e}}$ and $n_{\mathrm{e}} L$ in discharge $\# 1058164$ for $I_{\mathrm{RMP}}=0,3.2$ and $3.9 \mathrm{kA}$ at $0.30254,0.3317$ and $0.35792 \mathrm{~s}$, respectively. $n_{\mathrm{e}} L$ is obtained by a laser-based far-infrared polarimeter-interferometer system.

To understand the mechanism leading to region III, radial profiles of $A_{\mathrm{st}}, T_{\mathrm{e}}$ and $n_{\mathrm{e}} L$ are displayed in FIG. 2 for three cases, with $I_{\mathrm{RMP}}=0,3.2$ and $3.9 \mathrm{kA}$, corresponding to those without RMP, in region II and III, respectively. The value of $A_{\text {st }}$ is negative in the core $(-8 \mathrm{~cm}<r<11 \mathrm{~cm})$ but is positive outside. The inversion radii of sawtooth $(\sim \operatorname{radii}$ of $q=1$ surface) are at $-9 \mathrm{~cm}$ and $12 \mathrm{~cm}$ on the high and low field sides (HFS and LFS), respectively. The sawtooth magnitude $\left|A_{\mathrm{st}}\right|$ in region II slightly increases, compared to that without RMP. In region III with $I_{\mathrm{RMP}}=3.9 \mathrm{kA},\left|A_{\mathrm{st}}\right|$ is significantly reduced both inside and outside the inversion radii. $\left|A_{\mathrm{st}}\right|$ in the core is reduced by $50 \%$.

$T_{\mathrm{e}}\left(n_{\mathrm{e}} L\right)$ slightly increases (decreases by $2 \%$ ) in region II compared to that without RMP. In region III, $n_{\mathrm{e}} L$ decreases by $5 \%$ in the core, and $T_{\mathrm{e}}$ decreases more inside $q=1$ surface. There is a flattening of the $T_{\mathrm{e}}$ profile around $q=1$ surface in the mid-plane on both HFS and LFS. For the circular plasmas on J-TEXT, this flattening can be explained by the formation of a locked magnetic island with an even poloidal mode number due to the $2 / 2$ RMP penetration. 


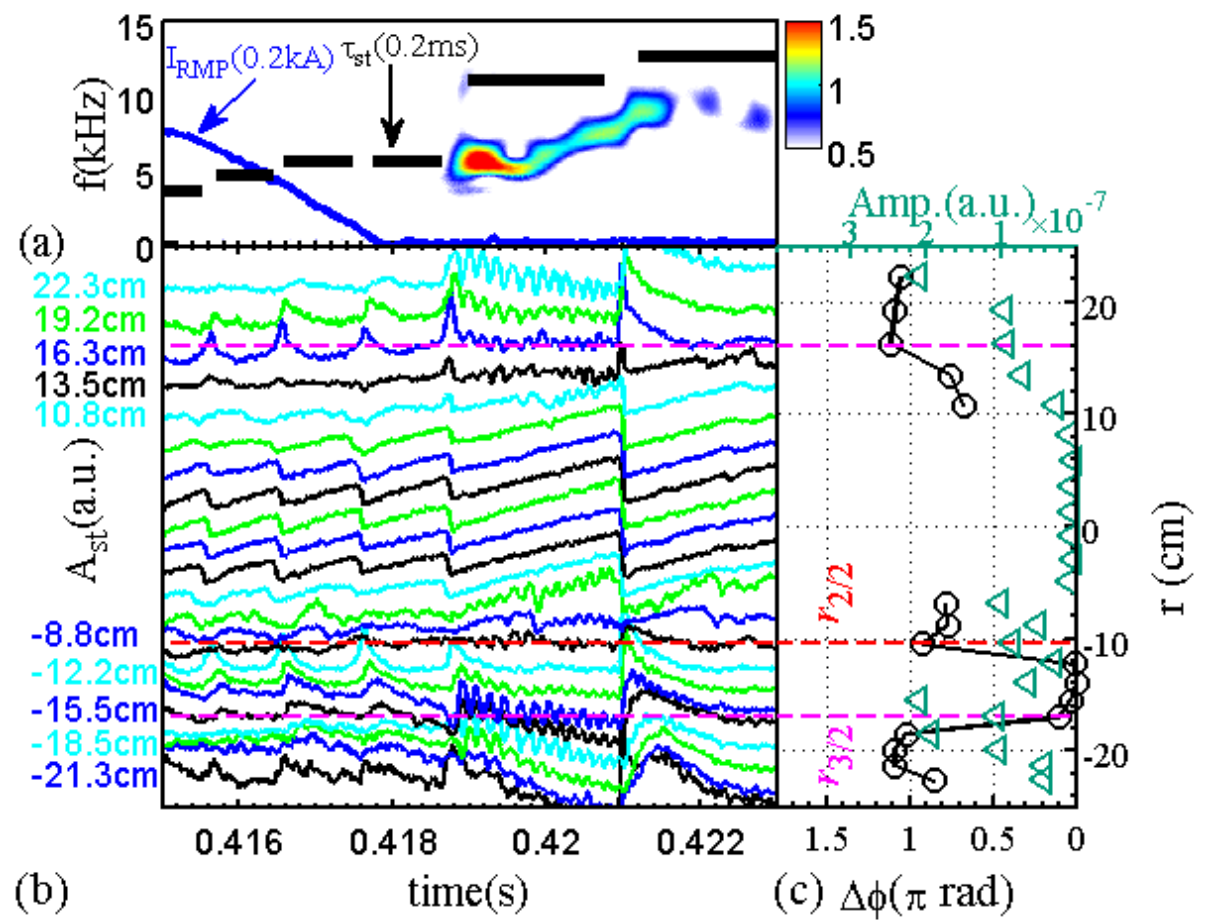

FIG. 3. Unlocking of $2 / 2$ and $3 / 2$ islands after the removal of $n=2$ RMP. (a) Frequency spectrum of the Mirnov probe signal, $I_{\mathrm{RMP}}$ and $\tau_{\mathrm{st}}$; (b) perturbed ECE signals, $\delta T_{\mathrm{e}} / T_{\mathrm{e}}$, from $0.415 \mathrm{~s}$ to $0.423 \mathrm{~s}$; (c) radial distribution of the phase (black circles) and amplitude (green triangles) of $\delta T_{\mathrm{e}} / T_{\mathrm{e}}$, obtained by the cross power spectrum analysis. The phase is not shown in the region with a rather low amplitude of $T_{\mathrm{e}}$ oscillation.

The excitation of $2 / 2$ locked island is confirmed by the unlocking of $2 / 2$ island, as shown in FIG. 3 . When $I_{\text {RMP }}$ is reduced to 0 around $0.419 \mathrm{~s}$ (FIG. 3(a)), high-frequency oscillations are observed from both magnetic and ECE signals (FIG. 3(b)), and they appear at a frequency about $5 \mathrm{kHz}$, spin up towards $10 \mathrm{kHz}$ later and then decay away within 3 ms. A dominant $m / n=3 / 2$ structure is found from $\mathrm{d} B_{\theta} / \mathrm{d} t$ signals measured from the toroidal and poloidal Mirnov probe arrays, indicating the unlocking of a 3/2 mode. Meanwhile, the $T_{\mathrm{e}}$ oscillations are observed from the ECE signals measured in the regions of $r \in[-20 \mathrm{~cm},-7 \mathrm{~cm}]$ and [ $9 \mathrm{~cm}, 22 \mathrm{~cm}]$ (FIG. 3(b)), covering both the $q=3 / 2$ and $2 / 2$ rational surfaces.

The mode structure is identified by the radial distribution of the phase and amplitude of $T_{\mathrm{e}}$ oscillation, as shown in FIG. 3(c). A clear reverse of the phase is found near $r=-18 \mathrm{~cm}$, which is around the $q=3 / 2$ surface. Hence, the existence of $3 / 2$ island is confirmed. Another phase reverse locates around $r=-11 \mathrm{~cm}$, which is near the outer boundary of the $T_{\mathrm{e}}$ profile flattening in the HFS (see FIG. 2(b)). In addition, the phases are almost the same for channels inside the $q=1$ surface on both HFS and LFS, such as those at $r=-8.8 \mathrm{~cm}$ and $10.8 \mathrm{~cm}$. This indicates an island with an even $m$, i.e. a $2 / 2$ island. The unlocking of the $2 / 2$ island proves the speculation of a $2 / 2$ locked island in region III. There is no clear phase reverse in the LFS for both the $2 / 2$ and $3 / 2$ islands, which is not yet understood clearly. In a toroidal plasma, the magnetic island width is smaller in the LFS than the HFS, while the spatial separation between adjacent ECE channels is larger in the LFS. Besides, the unlocked island is smaller than the locked island. These effects worsen the spatial resolution of ECE measurement compared the island size, which might lead to the lack of measured phase reversal in the LFS. Future 
measurement via ECE-Imaging [26] might provide further evidence of 2/2 and 3/2 islands on LFS.

A key connection between the $2 / 2$ island and sawteeth is that the sawtooth period recovers once the $2 / 2$ island unlocks and decays away. Summing the above observations up, it is concluded that $(a)$ the penetration of $n=2$ RMP excites locked $2 / 2$ and $3 / 2$ islands in region III and triggers the abrupt decrease of $\tau_{\mathrm{st}} ;(b)$ the $2 / 2$ and 3/2 islands unlock when RMP is off; (c) with the spinning-up and decay of the $2 / 2$ island, sawteeth recover.
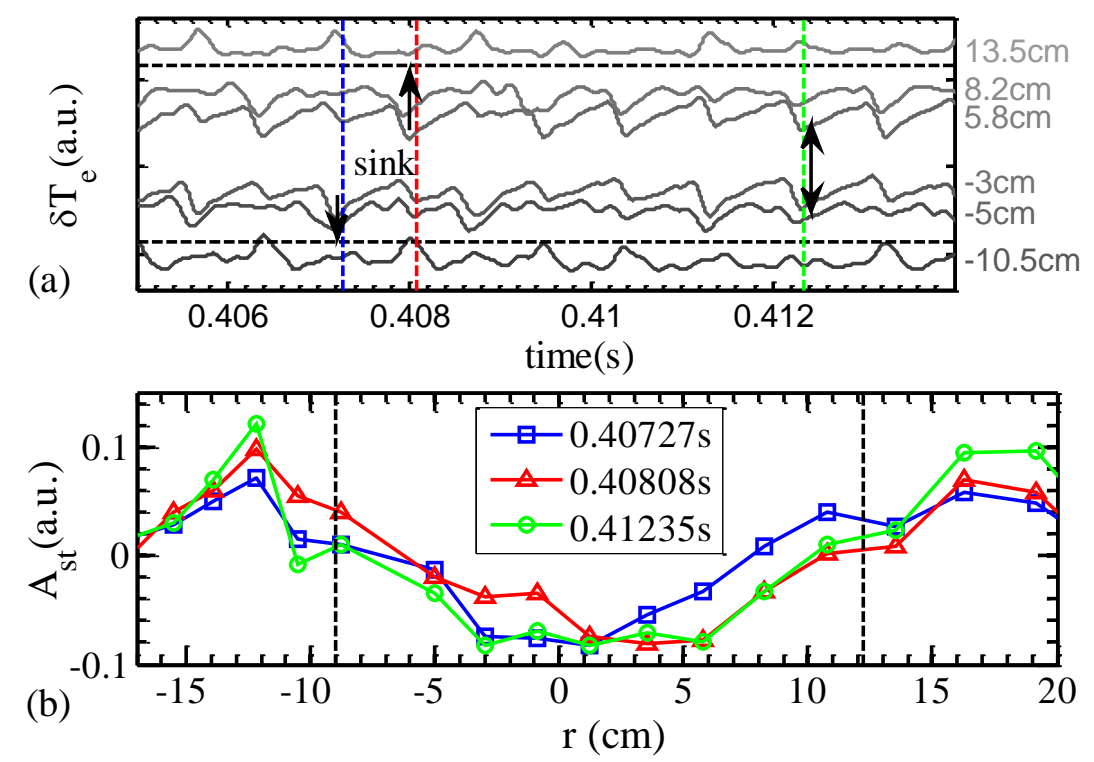

FIG. 4. Comparison of three sawtooth crashes at $0.303 \mathrm{~s}, 0.407 \mathrm{~s}$ and $0.408 \mathrm{~s}$ in region III. (a) The perturbed $T_{\mathrm{e}}$ near $r_{\text {inv }}$ in the LFS and HFS, (b) radial profile of sawtooth magnitude $A_{\text {st. }}$.

In the presence of the $2 / 2$ locked island in region III, not only $\tau_{\mathrm{st}}$ and $A_{\mathrm{st}}$ are reduced, but also the sawtooth crashes show new features. As marked by the dark arrow at about $0.407 \mathrm{~s}$ in FIG. 4(a), the sawtooth crash leads to a large $\Delta T_{\mathrm{e}}$ inside the inversion radius on the HFS but little on the LFS. The next crash shows the opposite: a large $\Delta T_{\mathrm{e}}$ on the LFS. They are compared in FIG. 4(b) by the radial distributions of $A_{\text {st }}$, termed as "HFS crash" and "LFS crash" respectively. The core of HFS and LFS crashes locate at $r=-1 \mathrm{~cm}$ and $3.5 \mathrm{~cm}$ on the mid-plane respectively, and their distance $(4.5 \mathrm{~cm})$ is much more than the spatial resolution (about $2 \mathrm{~cm}$ ) of ECE measurements. From $0.405 \mathrm{~s}$ to $0.415 \mathrm{~s}$, both HFS and LFS crashes happen frequently, as shown in FIG. 4(a). Since the ECE measurement is one dimensional, these asymmetries cannot be observed if the location of sawtooth crash is not mainly on the LFS or HFS, as might be the case for the sawtooth crash at $0.412 \mathrm{~s}$ with a large $\Delta T_{\mathrm{e}}$ on both the LFS and LFS.

Assuming heat transport of sawtooth crash across the reconnection layer near $q=1$ surface, the poloidal positions of sawtooth crashes (i.e. heat pulse), $\theta_{\text {crash }}$, can be estimated by soft X-ray emission in plasma core, simplistically by two SXR cameras viewing the plasma both horizontally from the mid-plane and vertically from the bottom. In plasma with a circular cross section, the displacement of the plasma core can be estimated by [27]

$$
\zeta_{\alpha} \approx \frac{\sum_{i} I_{s x r}^{\alpha, i} r_{\alpha, i}}{\sum_{i} I_{s x r}^{\alpha, i}}, \quad \alpha=R, Z, \quad\left|r_{\alpha, i}\right|<\left|r_{i n v}\right|,
$$

where $I_{s x r}^{\alpha, i}$ is the soft X-ray emission intensity, $\alpha$ represents the horizontal or vertical displacement for $R$ or $Z$ respectively, 
and $i$ is the $i^{\text {th }}$ SXR chord which locates inside the sawtooth inversion radius $r_{\text {inv }}$, i.e. $\left|r_{\mathrm{i}, \alpha}\right|<\left|r_{\text {inv }}\right|$, where $r_{\mathrm{i}, \alpha}$ is the chord radius of chord $i$. The displacement $\zeta_{\alpha}$ contains two terms caused by sharp crash and slow movement of the plasma core,

$$
\zeta_{\alpha}=\zeta_{\alpha}^{\text {slow }}+\zeta_{\alpha}^{\text {sharp }}
$$

The term from sharp crash $\zeta_{\alpha}^{\text {sharp }}$ of higher frequency can be extracted by a high-pass filter and hence $\theta_{\text {crash }}$ is obtained by the sharp displacements due to sawtooth crash,

$$
\theta_{\text {crash }}=f\left(\zeta_{R}^{\text {sharp }}, \zeta_{Z}^{\text {sharp }}\right),
$$

where $f$ is a transformation function from $(R, Z)$ coordinate to $(\theta, r)$ coordinate.

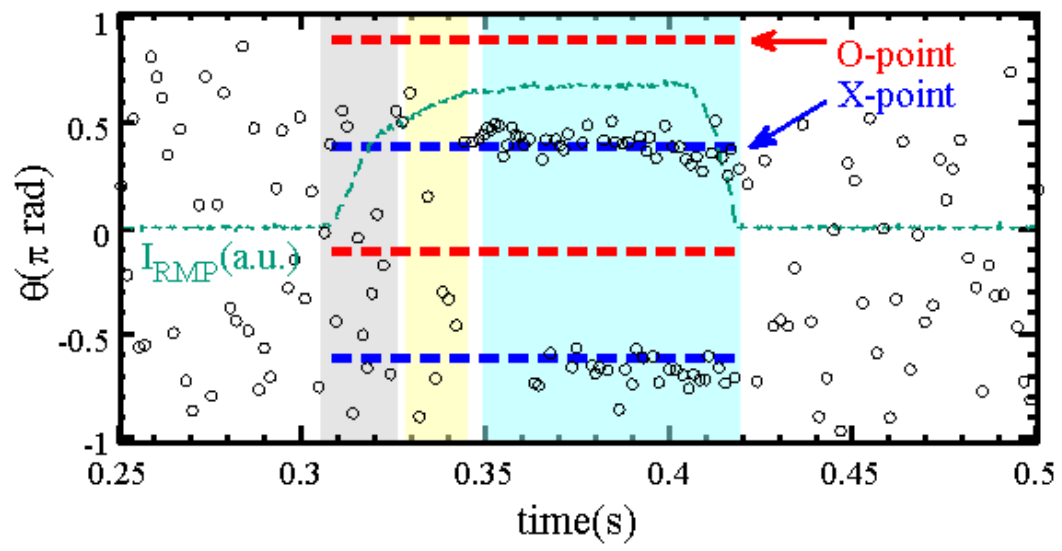

FIG. 5. Poloidal positions of sawtooth crashes (black circles) are compared to that of the $2 / 2$ locked island (red and blue dashed lines for $\mathrm{O}$ - and X-points respectively). The noise error is $\pm 0.16 \pi$, coming mainly from noises and interferences in SXR signals. The $2 / 2$ locked island phases are calculated from the RMP phase using vacuum assumption. The shaded areas represent region I, II and III.

The poloidal positions of sawtooth crashes are displayed in FIG. 5. It is found that the crashes are at a random poloidal location without RMP, in region I and II, while $\theta_{\text {crash }}$ is around $0.4 \pi$ or $-0.6 \pi$ in region III. Adopting the vacuum assumption, the phases of the $2 / 2$ locked islands are determined by the $2 / 2$ RMP, which are $-0.12 \pi$ and $0.88 \pi$ for the O-points (two red dashed lines), $-0.62 \pi$ and $0.38 \pi$ for the X-points (blue dashed) at the toroidal location of the SXR arrays. Hence in region III, the heat transport crosses the X-points of the locked $2 / 2$ islands, similar to the heat transport across the X-points of $2 / 2$ island after sawtooth crashes observed on HT-7 [28]. No rotating precursor 1/1 modes are detected before sawtooth crashes in region III, so that the crashes might be induced by the growth of $1 / 1$ modes locked to $2 / 2$ islands at one of the two phases

FIG. 6 displays the sawtooth response to 2/2 RMPs in the $\left(n_{\mathrm{e}}, q_{\mathrm{a}}\right)$ plane for more than 60 discharges with the plasma current $195-210 \mathrm{kA}$ and toroidal magnetic field 1.75T. There is little change of sawtooth behavior in the high $q_{\mathrm{a}}$ or high $n_{\mathrm{e}}$ region, while the sawtooth mitigation is observed in region III with lower $q_{\mathrm{a}}$ and $n_{\mathrm{e}}$ values $\left(q_{\mathrm{a}}<2.8\right.$ and $n_{\mathrm{e}}<2 \times 10^{19} \mathrm{~m}^{-}$ ${ }^{3}$ ). The dependence of sawtooth response to RMPs on $n_{\mathrm{e}}$ and $q_{\mathrm{a}}$ is similar to that of RMP penetration. The RMP penetration threshold is generally observed to decrease with $q_{\mathrm{a}}$ and $n_{\mathrm{e}}$ [29-30]. Hence, $I_{\mathrm{th}}$ decreases from the upper-right towards the lower-left in the $n_{\mathrm{e}}-q_{\text {a }}$ plane in FIG. 6 , and $I_{\mathrm{th}}$ might decrease to a value below $I_{\mathrm{RMP}}$ at the boundary between region II and III, leading to the penetration of $2 / 2 \mathrm{RMP}$ and a decrease of sawtooth magnitude in region III. With the increase of $I_{\mathrm{RMP}} / I_{\mathrm{th}}$, region I, II and III appear successively from left to right in FIG. 1 (with fixed $I_{\text {th }}$ ) and from upper-right towards the lower- 
left in FIG. 6 (with fixed $I_{\mathrm{RMP}}$ ). It is therefore speculated that the sawtooth amplitude reduction can be achieved for $q_{\mathrm{a}}>3$ if a larger RMP is available.

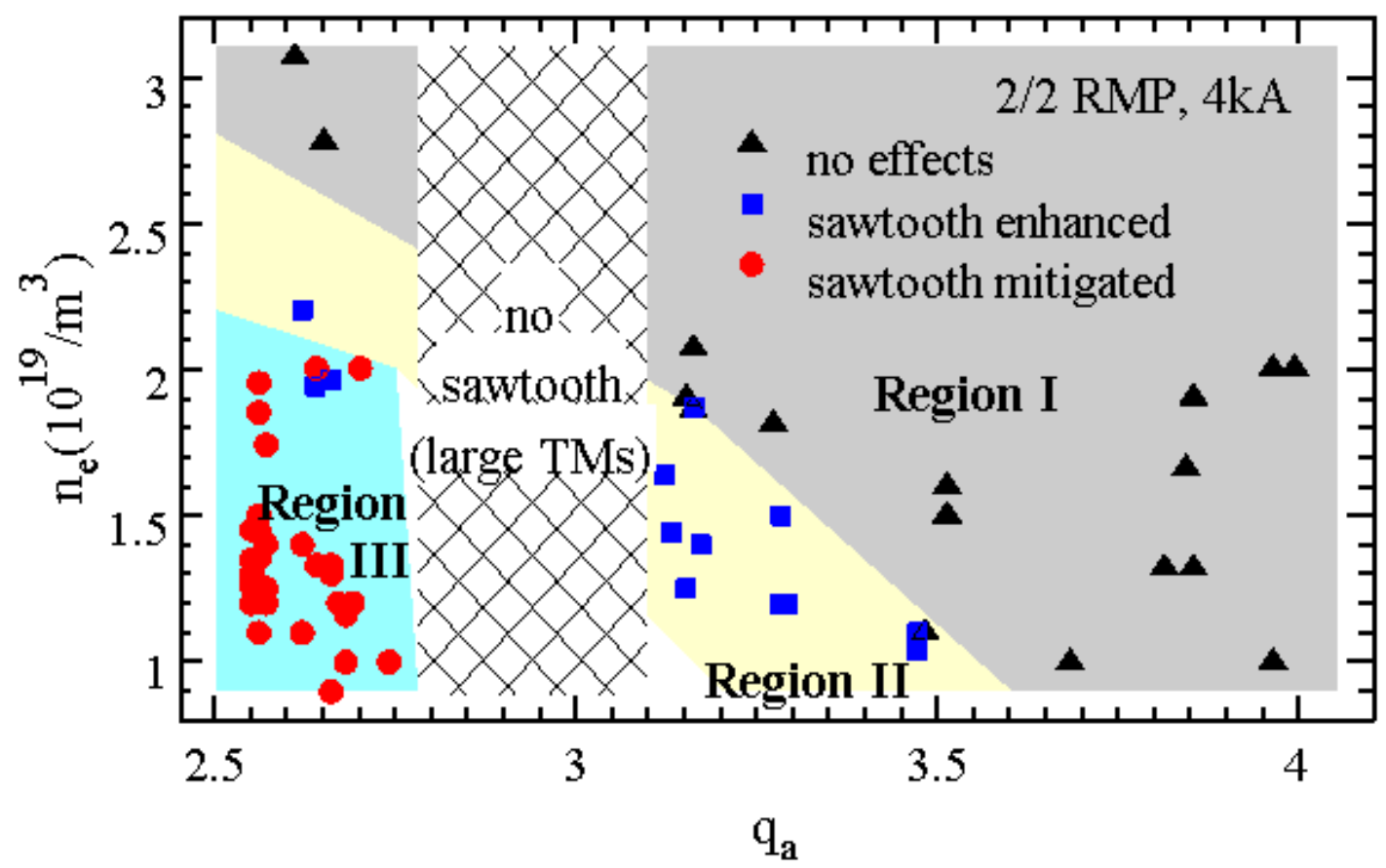

FIG. 6. Sawtooth response to $2 / 2$ RMPs in the $n_{\mathrm{e}}-q_{\text {a }}$ plane with $I_{\mathrm{RMP}}=4 \mathrm{kA}$. There are 17 discharges with no clear change of sawtooth behavior (black triangles, regions I), 14 discharges with a small increase of sawtooth amplitude (blue squares, regions II) and 33 discharges with significant decrease of sawtooth amplitude (red circles, region III). The region with $q_{\mathrm{a}} \in[2.8,3.1]$ is excluded due to the presence of large tearing modes and hence no sawtooth oscillations.

\section{Discussion and summary}

It should be mention that our successful sawteeth mitigation experiments are limited to a narrow parameter regime of the J-TEXT experiment, as shown in FIG. 6. As the penetration of the 2/2 RMP is the key for sawteeth mitigation, FIG. 7 displays the dependence of the penetration threshold of the 2/2 RMP (in terms of the RMP coil current $I_{\mathrm{th}}$ ) on electron density in 11 successive discharges (\#1058160 to \#1058170), with $n_{e}$ in the range of $1-1.6 \times 10^{19} \mathrm{~m}^{-3}, I_{\mathrm{p}} \sim 210 \mathrm{kA}$ and $B_{\mathrm{t}}$ $\sim 1.75 \mathrm{~T}$. The threshold for the 2/2 RMP penetration increase with $n_{e}$. This indicates that for higher plasma density, large RMPs are required for sawteeth mitigation. Extrapolating from a linear fitting shown in FIG. 7, the 2/2 RMP penetration threshold is around $5.7 \mathrm{kA}$ on J-TEXT for $n_{e}=3 \times 10^{19} \mathrm{~m}^{-3}$. After the installation of 12 new two-turns RMP coils on JTEXT in 2017 [18], however, the DC power supply (PS) of the RMP system can only provide the maximal current of 4 kA to all the 24 in-vessel RMP coils (12 old one-turn and 12 new two-turns coils). An upgrade of the PS is required in order to further carry out experiments at high plasma density. 


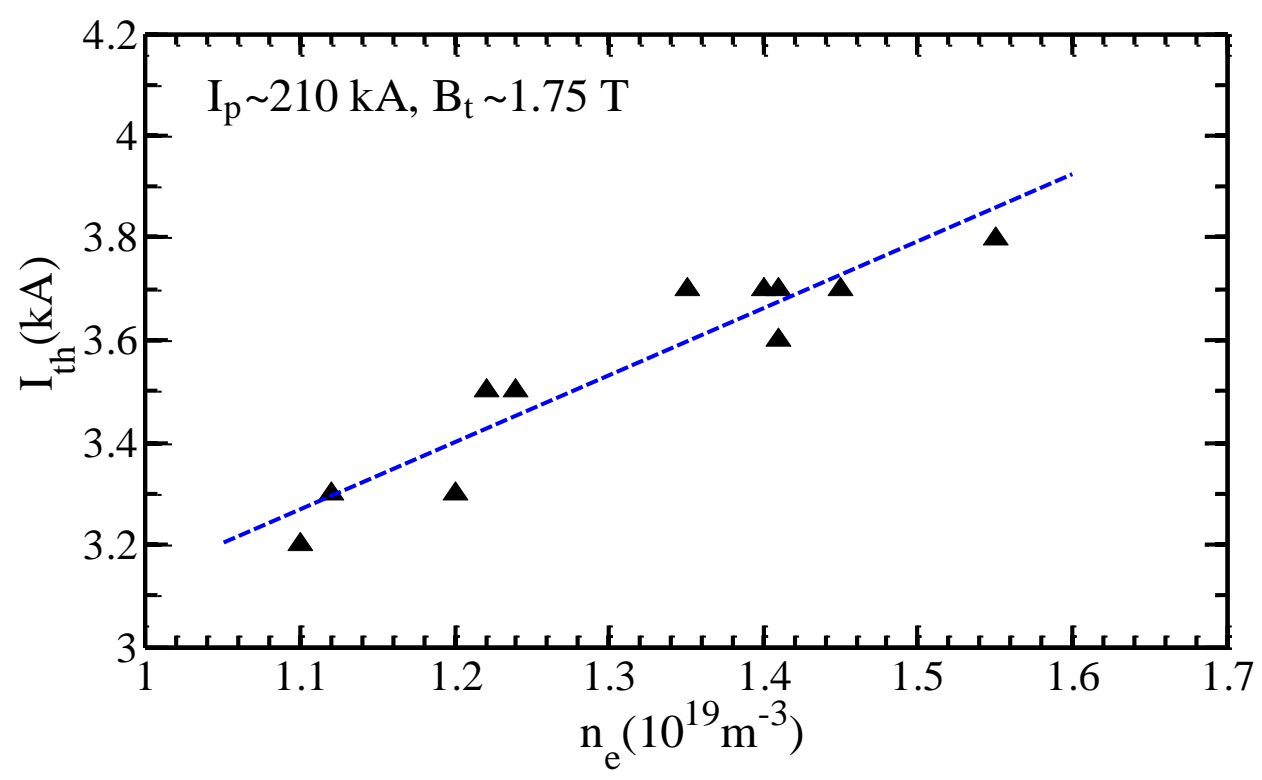

FIG. 7. The threshold of the $2 / 2$ RMP penetration as a function of electron density, with $I_{\mathrm{p}} \sim 210 \mathrm{kA}$ and $B_{\mathrm{t}} \sim 1.75 \mathrm{~T}$. The linear fitting to the experimental data is shown by the blue dashed line, leading to $I_{\mathrm{RMP}}[\mathrm{kA}]=1.3 \times n_{e}\left[10^{19} \mathrm{~m}^{-3}\right]+1.8$.

On the other hand, it is known from two-fluid theory and simulations that the RMP penetration threshold is mostly determined by the local electron fluid velocity at the resonant surface [31-32], as found in experiments [33]. Although no theoretical simulations has been performed for the 2/2 RMP yet, the earlier theoretical results should also be valid for our $m=2$ case. The scaling of the penetration threshold with the electron density shown in FIG. 7 could be due to the different local plasma rotation and diamagnetic drift frequency for different electron density [20]. Measurement of the local plasma rotation velocity are required in order to compare the experimental results with theoretical ones for a better understanding of the penetration threshold.

For ITER or a fusion reactor, the plasma rotation velocity is expected to be low due to low torque input, so that the required 2/2 RMP for penetration could be smaller comparing to existing tokamaks with high power NBI injection. As existing theories indicate that the local electron fluid velocity is the most important parameter in determining the RMP penetration [31-32], by adjusting the NBI direction or heating method to have a small local electron fluid velocity at $q=1$ surface, the sawteeth mitigation by $2 / 2$ RMP could be possible in moderate-sized tokamaks, if the $2 / 2$ component RMP is sufficiently large.

In our experiments, no $2 / 1$ mode has been triggered by the $2 / 2$ RMPs. There will be always a $2 / 1$ component in experiments, since no RMP system is perfect. For even number of toroidal sets of RMP coils, the $n=1$ component due to the imperfection of RMP coils is quite small when operating at $n=2$ configuration. For example, the RMP coil at the LFS of Port 12 on the J-TEXT has only half the length in toroidal direction than other LFS coils, which leads to a 2/1 component of 0.5 Gauss/kA, being 15 times smaller than that of the 2/2 component. For the expected penetration threshold of $2 / 2$ RMP, the $2 / 1$ component would be around 1.7 or 2.8 Gauss for $n_{e}=1.5$ or $3 \times 10^{19} \mathrm{~m}^{-3}$, while the $2 / 1$ RMP penetration thresholds are around 9 and 14 Gauss respectively [20]. The residual 2/1 RMP field is only 1/5 of the penetration threshold.

For RMP coils with odd number of sets in toroidal direction, e.g. in DIII-D or ITER, the $n=1$ component could be large 
in the $n=2$ dominate configuration. In these cases, either a compensation of the $n=1$ component or the operation with a dominant 3/3 RMP would be beneficial. As the effect of the 3/3 RMP on sawteeth is expected to be similar to that of the 2/2 RMP, it could be a safer choice for controlling sawteeth in larger size tokamaks.

Our work is the first experimental demonstration that RMP $(2 / 2)$ can mitigate sawteeth with $q_{a}>2$. These experiments are, however, only in Ohmic discharges with relatively low plasma density and temperature. After the installation of 500 kW ECRH system on J-TEXT together with the upgrade of power supply system [34], the sawteeth mitigation can be extended to higher plasma density and temperature regime. The sawteeth in current J-TEXT experiments have shorter periods and smaller amplitudes than those of larger tokamaks with auxiliary heating, where the sawtooth period reduction might not be necessarily associated with a reduction of the amplitude, and NTM triggering was found to be correlated with the sawtooth period rather than its amplitude [35]. As the sawtooth mitigation by RMPs relies on the field penetration and the formation of $m>1$ magnetic island at $q=1$ surface, which changes the local plasma parameters around and inside the $q=1$ surface, this mechanism is expected to also work for high density and temperature plasmas, if the $2 / 2$ or $3 / 3$ RMP is sufficiently large to be penetrated in.

The core plasma rotation is found to be driven towards the co-current direction by RMPs with a velocity change about 14 km/s even in Region II, as shown in FIG. 1(c), being consistent with earlier theoretical prediction [36]. This implies another possible application of the 2/2 (or 3/3) RMP: to drive core plasma rotation in a fusion device. It is well known that plasma rotation is important for plasma confinement and stability, while the available input torque is low in a fusion reactor.

In conclusion, the reduction of sawtooth magnitude is demonstrated by a novel and simple method, i.e. applying the $2 / 2$ RMP. The penetration of RMPs and formation of $2 / 2$ locked island are found to be related to the reduction of sawtooth magnitude, revealing a possible new way, i.e., using RMPs with $m / n=1$ but $m, n>1$, for controlling sawteeth.

\section{Acknowledgements}

The authors are very grateful for the help of J-TEXT team. This work is supported by the National MCF Energy R\&D Program of China (Contract No. 2018YFE0309100), State Key Laboratory of Advanced Electromagnetic Engineering and Technology (Grant No. AEET2018KF006), the International Partnership Program of Chinese Academy of Sciences (No. Y16YZ17271) and the National Natural Science Foundation of China (51821005). The Shenzhen Clean Energy Research Institute is appreciated.

\section{References}

[1] Von Goeler S., Stodiek W. and Sauthoff N. 1974 Phys. Rev. Lett. 331201.

[2] Kadomtsev B. B. 1975 Fiz. Plazmy 1710.

[3] Sykes A. and Wesson J. A. 1976 Phys. Rev. Lett. 37140.

[4] Edwards A.W. et al 1986 Phys. Rev. Lett. 57210.

[5] Sauter O. et al 2002 Phys. Rev. Lett. 88105001.

[6] ITER Physics Basis 1999 Nucl. Fusion 392137. 
[7] Söldner F. X. et al 1986 Phys. Rev. Lett. 571137.

[8] Hanada K. et al 1991 Phys. Rev. Lett. 661974.

[9] Sauter O. et al 2002 Phys. Rev. Lett. 88105001.

[10] Eriksson L.-G. et al 2004 Phys. Rev. Lett. 92235004.

[11] Compbell D. J. et al 1988 Phys. Rev. Lett. 602148.

[12] Chapman I. T. et al 2006 Nucl. Fusion 461009.

[13] Zohm H. et al 2015 Nucl. Fusion 55104010.

[14] Klein A. J. et al 2005 Phys. Plasmas 12040703.

[15] Evans T. E. et al 2004 Phys. Rev. Lett. 92235003.

[16] Piron C. et al 2016 Nucl. Fusion 56106012.

[17] Yu Q., Günter S. and Lackner K. 2014 Nucl. Fusion 54072005.

[18] Liang Y. et al 2019 Nucl. Fusion 59112016.

[19] Rao B. et al 2014 Fusion Eng. Des. 89378.

[20] Huang Z. et al 2020 Nucl. Fusion 60064003.

[21] Yang Z. et al 2016 Rev. Sci. Instrum. 87 11E112.

[22] Jin W. et al 2012 Rev. Sci. Instrum. 83 10E502.

[23] Chen J. et al 2014 Rev. Sci. Instrum. 85 11D303.

[24] Guo D. et al 2017 Rev. Sci. Instrum. 88123502.

[25] Li J. et al 2014 Rev. Sci. Instrum. 85 11E414.

[26] Pan X. M. et al 2016 Rev. Sci. Instrum. 87, 11E106.

[27] Ding Y. H. et al 2009 Nuclear Instruments \& Methods in Physics Research A 606743.

[28] Sun Y. et al 2009 Plasma Phys. Control. Fusion 51065001.

[29] Buttery R. J. et al 1999 Nucl. Fusion 391827.

[30] Lanctot M. J. et al 2017 Phys. Plasmas 24056117.

[31] Yu Q., Günter S., Kikuchi Y. and Finken K.H. 2008 Nucl. Fusion 48, 024007.

[32] Yu Q. and Günter S. 2011 Nucl. Fusion 51073030.

[33] Koslowski H.R., Liang Y., Krämer-Flecken A. et al 2006 Nucl. Fusion 46, L1.

[34] Chen X. X. et al., Fusion Engineering and Design, submitted.

[35] Chapman I. T. et al 2010 Nucl. Fusion 50102001.

[36] Yu Q., Günter S. and Finken K. H. 2009 Phys. Plasmas 16042301. 